\title{
Composición fisicoquímica de la semilla y del aceite de la semilla del Canime (Copaifera officinalis $L$ )
}

\author{
Jennifer J. Lafont ${ }^{\star}$, Manuel S. Páez y Edineldo Lans \\ Universidad de Córdoba, Facultad de Ciencias Básicas e Ingeniería, Departamento de Química, \\ Carrera 6 No 76-103, Montería, Córdoba-Colombia (e-mail: jenniferlafontm@yahoo.es) \\ *autor a quien debe ser dirigida la correspondencia
}

Recibido Jul. 20, 2010; Aceptado Oct. 06, 2010; Versión Final recibida Nov. 26, 2010

\section{Resumen}

Se han determinado las características morfológicas, composición proximal y cantidad de minerales presentes en la semilla de canime (Copaifera officinalis $L$ ) y de su aceite. También se han determinado algunas características fisicoquímicas y el perfil de ácidos grasos, no reportados aún en la literatura. Las semillas fueron trituradas el aceite fue obtenido por extracción soxhlet con diferentes solventes para encontrar el de mejor rendimiento. Se analizó: índice de yodo, acidez, puntos de fluidez, enturbiamiento y composición química mediante cromatografía de gases acoplada a masas. Los resultados muestran que las semillas de canime contienen nutrientes considerados principales en la elaboración de alimento para animales, como carbohidratos, ácidos grasos y vitamina E. Se recomienda el aprovechamiento de estas semillas para uso como alimento para animales.

Palabras claves: Copaifera officinalis L, Almendra de canime, Análisis proximal, Perfil de ácidos grasos.

\section{Physicochemical composition of the seed and oil of Canime seed (Copaifera officinalis $L$ )}

\begin{abstract}
The morphological characteristics, the proximate composition and the quantity of minerals present in the seed of canime (Copaifera officinalis $L$ ) and its oil were determined. Also, some physicochemical characteristics and fatty acid profile, not yet reported in the literature, were determined. The seeds were crushed and the oil was extracted by soxhlet using different solvents to find that giving the optimum yield. lodine index, acidity, fluidity, turbidity and chemical composition through chromatography of gas joined to masses, were determined. The results show that the canime seeds contain nutrients that are considered of special importance for making animal food, such as carbohydrates, fatty acids and vitamin E. For all this, the use of these seeds is recommended for manufacturing animal food.
\end{abstract}

Keywords: Copaifera officinalis L, Almond canıme, Proximate analysis, Fatty-acid profile. 


\section{INTRODUCCIÓN}

El canime o copaiba (Copaifera officinalis $L$ ), es un árbol de tronco recto con superficie rugosa que puede alcanzar entre 20 y 30 metros de altura, sus flores son blancas, pequeñas, bisexuales y olorosas, su fruto es dehiscente en dos valvas de color marrón; las semillas son ovoides y pequeñas, de color negro, se encuentran de 1 a 2 envueltas en un arilo de color amarillo; este árbol es originario de Sur América pero también se encuentra en Puerto Rico y Hawaii, es propio de climas tropicales húmedos y secos, de cuya madera se construyen embarcaciones (Silva y Vieira, 2008). El Copaifera officinalis $L$, es un árbol que pertenece a la familia Caesalpiniaceae y al genero Copaifera; existen cerca de 70 especies de este genero (Chen et al, 2009), las cuales han sido utilizadas ampliamente como árbol maderable y como productor de aceite extraído de la resina del tronco que ha sido utilizado como fijador de aromas en perfumes y jabones (Sant'Anna et al; 2007) y en la medicina tradicional como antiiflamatorio y analgésico (Matos et al., 2007; Veiga, 2007), antiséptico y cicatrizante de heridas (Brito et al., 2005), con acción citotóxica y anticancerosa (Matos et al., 2008), actividad antimicrobiana (Santos et al., 2008; Freitas y Sousa, 2007), entre otras.

El género Copaifera officinalis $L$, se encuentra en la costa norte de Colombia, específicamente en el departamento de Córdoba, su semilla cae abundantemente del árbol y no es provechada en ninguna forma; en la literatura se encuentran muy pocos estudios acerca de esta especie los cuales están enfocados al análisis de la oleorresina de su tronco (Chen et al, 2009) y no se ha encontrado información científica acerca de las semillas y el aceite de las mismas; esta fue la razón que nos motivó a evaluar las características morfológicas, la composición proximal y la cantidad de minerales presentes en la semilla del canime; como también determinar las características fisicoquímicas y perfil de ácidos grasos del aceite, con el fin de encontrar una posible utilidad.

\section{METODOLOGÍA}

Las semillas de Canime (Copaifera officinalis $L$ ), fueron recolectadas en el municipio de Tierralta Córdoba, (Colombia); se sometieron a calentamiento en una mufla a $150^{\circ} \mathrm{C}$ durante 1.5 horas, posteriormente se les desprendió la almendra de la cáscara, con golpes suaves. De las almendras así obtenidas se seleccionó una muestra de 30 semillas, las cuales fueron clasificadas en tres grupos de 10, como grandes, medianas y pequeñas, a fin de determinar sus características morfológicas (largo, ancho, espesor y peso), con la ayuda de un Nonio o vernier $( \pm 0.01 \mathrm{~cm})$ y una balanza analítica OHAUS $\left( \pm 1 \times 10^{-4} \mathrm{~g}\right)$ de precisión respectivamente. Posteriormente, las almendras fueron trituradas utilizando un mortero de porcelana; los gránulos así obtenidos se empacaron en bolsas de poliestireno con cierre hermético y se almacenaron en la oscuridad a temperatura ambiente; siendo esta la materia prima empleada para realizar los análisis de humedad, cenizas, proteínas, grasa cruda, fibra, carbohidratos, análisis de minerales y extracción de aceites. Todos los procedimientos se realizaron por triplicado; con el propósito de evaluar el valor promedio $(x)$ y la desviación estándar $(\sigma)$ para cada análisis. Para determinar el contenido de humedad (Amin et al., 2007) las almendras trituradas se pesaron y se vertieron en un crisol, luego se introdujeron en una mufla $\mathrm{E} \& Q$ equipada con una termocoupla tipo $\mathrm{K}$, a $100^{\circ} \mathrm{C}$ durante 2 horas para retirar la humedad presente; transcurrido este tiempo, se retiraron de la mufla, y se dejaron enfriar en un desecador, luego se pesaron y se calculó el contenido de humedad con la diferencia de peso. El contenido de cenizas (C) (Amin et al., 2007) se determinó secando la muestra en una mufla a $100^{\circ} \mathrm{C}$ durante 2 horas, después se pesó $\left(\mathrm{W}_{\mathrm{M}}\right)$ y se calcinó a $550^{\circ} \mathrm{C}$ hasta peso constante $(6$ horas); las cenizas así obtenidas se pesaron $\left(\mathrm{W}_{\mathrm{C}}\right)$ y se calculó el contenido de cenizas usando la ecuación 1.

$C=\left(W_{C} / W_{M}\right)^{*} 100 \%$

El contenido de proteínas (P) (Henríquez, et al., 2008) se determinó por el método Kjeldahl; el proceso se inicia con la digestión que se obtiene al mezclar $0.2473 \mathrm{~g}$ de la muestra con $10 \mathrm{~mL}$ ácido sulfúrico concentrado mas el catalizador (mezcla de sulfato de cobre - sulfato de potasio), 
luego el sistema se sometió a un proceso de calentamiento y agitación durante durante 1.5horas. Posteriormente, se dejó reposar y se adicionaron lentamente $30 \mathrm{~mL}$ de agua y $80 \mathrm{~mL}$ de $\mathrm{NaOH}$ hasta completar $4 \mathrm{~min}$, finalmente la mezcla se destila dejando el terminal del equipo de destilación inmerso en una solución de acido bórico al $4 \% \mathrm{v} / \mathrm{v}$ el destilado obtenido en esta solución se titula con $\mathrm{H}_{2} \mathrm{SO}_{4} 0.0206 \mathrm{~N}$. El porcentaje de proteína se determinó mediante la ecuación 2; donde $\mathrm{V}$ : es el volumen en $\mathrm{mL}$ gastado de ácido en la titulación, $\mathrm{N}$ : representa la normalidad del ácido sulfúrico, 14: son los equivalentes-gramo del nitrógeno, $f$ es el factor proteico en este caso es de 6.25 y W es el peso de la muestra en gramos.

$P=\left[\left(V^{*} N^{*} 14^{*} f\right) /\left(100^{*} W_{M}\right)\right]^{*} 100 \%$

El contenido de fibra total (F) (Alvis; et al, 2008) se determinó mezclando $2 \mathrm{~g}$ de la muestra de almendras trituradas $\mathrm{W}_{\mathrm{M}}$ con $200 \mathrm{~mL}$ de ácido sulfúrico al $1.25 \% \mathrm{v} / \mathrm{v}$ y sometiéndola a ebullición durante 30 minutos, posteriormente se somete a filtración, después se lava con agua hirviendo varias veces, hasta obtener agua de lavado libre de ácido. El residuo se agrega en un beacker y se adiciona $200 \mathrm{~mL}$ de solución acuosa caliente de hidróxido de sodio al $1.25 \% \mathrm{p} / \mathrm{v}$, se deja hervir durante $30 \mathrm{~min}$ y se procede a filtrar, se lava varias veces con agua hirviendo y con porciones pequeñas de alcohol para recoger el residuo; que posteriormente es secado a $105^{\circ} \mathrm{C}$ durante 2 horas, se pesa y se registra como peso1 $\left(p_{1}\right)$; luego se lleva a la mufla a $500^{\circ} \mathrm{C}$ durante 1 hora y se pesa obteniendo el peso $2\left(p_{2}\right)$; el contenido de fibra se determina mediante la ecuación 3 .

$F=\left[\left(p_{1}-p_{2}\right) / W_{M}\right]^{*} 100 \%$

El contenido de lípidos o de aceite se determinó usando el método de reflujo con Soxhlet utilizando tres solventes diferentes para cada caso: hexano (Merck; 99.0\%), éter de petróleo (Merck, 99.0\%) y acetato de etilo (Merck, 99.5\%) siguiendo la metodología propuesta por (Asogwa et al., 2007); cada solvente se trabajó a sus temperaturas de ebullición así: hexano $\left(67^{\circ} \mathrm{C}\right)$, éter de petróleo $\left(57^{\circ} \mathrm{C}\right)$ y acetato de etilo $\left(45^{\circ} \mathrm{C}\right)$; con el fin de obtener un mayor rendimiento, en todos los casos se utilizó $100 \mathrm{~g}$ de muestra de almendras trituradas. El contenido de carbohidratos se calculó como la diferencia de 100 con la sumatoria de los valores de proteína cruda, lípidos, fibra y cenizas (Yookyung et al., 2007). La harina sin grasa, sobrante de la extracción de aceite por solventes a las almendras molidas, se le analizó el contenido de humedad, proteínas y cenizas por los métodos anteriores.Los análisis de metales $(\mathrm{K}, \mathrm{Cu}, \mathrm{Mg}, \mathrm{Zn}, \mathrm{Na})$ se realizaron utilizando un espectrofotómetro de absorción atómica marca Thermo Electron Corporation Modelo: S4AA System serie 6E711397. Para realizar este análisis, fue necesario preparar la siguiente solución: se tomó $5 \mathrm{~g}$ de muestra de almendras trituradas, las cuales fueron calcinadas siguiendo el procedimiento del contenido de cenizas, descrito anteriormente, las cenizas resultantes fueron disueltas en $3 \mathrm{~mL}$ de ácido nítrico concentrado y posteriormente diluídas con agua hasta obtener $25 \mathrm{~mL}$. Esta solución fue tomada para analizar el contenido de metales en el equipo (Amin et al., 2007). El límite de cuantificación (LC) del método estuvo en el rango de (0.03-0.09) mg/L, la exactitud $(R)$ entre (112 y 92.3) \% y la precisión (RSD) en el rango de (1.26-5.31) \%. El control de calidad analítica se realizó utilizando el estándar certificado titrisol-Merck $(1000 \mathrm{mg})$ y se hizo la lectura del estándar a cada 12 muestras, para verificar que las mediciones estuvieran dentro del rango establecido.

El aceite fue extraído por el método de soxhlet con diferentes solventes: acetato de etilo, éter de petróleo y n-hexano, con el fin de encontrar el mejor rendimiento; una vez obtenido el aceite se les realizó los índices de acidez y yodo, puntos de fluidez y de enturbamiento; en cuanto al índice de acidez (Hernández et al 2007) se tomó $1 \mathrm{~g}$ de muestra del aceite extraído con solvente y se tituló con una solución estándar de hidróxido de sodio $0.1 \mathrm{~N}$ en presencia de fenolftaleína, la muestra se disolvió con $40 \mathrm{~mL}$ de solución etanol-acetato de etilo 1:1, igualmente se titulo el blanco; los ácidos grasos libres se expresan como acido oleico. En el índice de yodo, se pesaron $0.8 \pm 0.0001 \mathrm{~g}$ de aceite y se disolvió en $15 \mathrm{~mL}$ de tetracloruro de carbono, luego se le agregaron $25 \mathrm{ml}$ del reactivo de Wijs y se dejó reposar la solución en la oscuridad, posteriormente se le agregó solución de yoduro de potasio y tituló con solución estándar de tiosulfato de sodio $0.02 \mathrm{~N}$, luego se adicionó almidón y se continuó titulando hasta que el color azul desapareciera. En el punto de fluidez método (ASTM D-97; 1996) se tomó $1 \mathrm{~mL}$ de muestra de aceite y se sometió a enfriamiento 
mediante un baño de agua con hielo y sal, se agitó suavemente la muestra sin interrupción durante el enfriamiento y se registró la temperatura mínima a la que se apreció movimiento de la sustancia. Para medir el punto de enturbiamiento (ASTM D-2500; 1999) se tomó $1 \mathrm{~mL}$ de muestra de aceite y se realizó el mismo procedimiento anterior, se registró la temperatura a la cual fue observada la primera nube de cristales o turbidez con la ayuda de una lupa, estos procedimientos se realizaron por triplicado. La composición química del aceite en estudio se realizó utilizando un cromatógrafo de gases, con detector selectivo de masas, Modelo Zebron con temperatura máxima de la columna $340 \mathrm{C}$, volumen de inyección $0.2 \mu \mathrm{L}$; temperatura del inyector $350 \mathrm{C}$ y tiempo total de corrida igual a $60 \mathrm{~min}$.

\section{RESULTADOS Y DISCUSIÓN}

En la tabla 1, se reportan las características morfológicas de la almendra del Copaifera officinalis $L$; mediante el valor promedio $(\mathrm{x})$ y la desviación estándar $(\sigma)$ del largo, ancho y espesor para tres tamaños diferentes, usando muestras de 10 semillas en cada caso y se encontró que presentaron mayor diferencia en el largo entre almendras grandes y pequeñas siendo de $0.33 \mathrm{~cm}$; en cuanto al ancho la diferencia fue de $0.27 \mathrm{~cm}$ y en el espesor de $0.19 \mathrm{~cm}$; indicando un mayor crecimiento longitudinal. Los valores de la desviación estandar indicados en la tabla 1 son pequeños se encuentran entre 0.02 y 0.18 ; los cuales indican el grado de dispersion de los datos con respecto al valor promedio; es decir a menor dispersion mayor es la precision en los datos obtenidos.

Tabla 1: Caracteristicas morfológicas de la semilla de canime

\begin{tabular}{lccc}
\hline Tamaño & $\begin{array}{c}\text { Largo/cm } \\
\mathrm{X} \pm \sigma\end{array}$ & $\begin{array}{c}\text { Ancho/cm } \\
\mathrm{X} \pm \sigma\end{array}$ & $\begin{array}{c}\text { Espesor/cm } \\
\mathrm{X} \pm \sigma\end{array}$ \\
\hline Grandes & $1.62 \pm 0.18$ & $1.23 \pm 0.10$ & $0.91 \pm 0.02$ \\
Medianas & $1.43 \pm 0.10$ & $1.20 \pm 0.12$ & $0.85 \pm 0.06$ \\
Pequeñas & $1.29 \pm 0.12$ & $0.96 \pm 0.10$ & $0.72 \pm 0.18$ \\
\hline
\end{tabular}

Los valores promedios de porcentajes y desviacion estandar del análisis proximal de la almendra y de la harina se registran en la tabla 2, de estos se infiere que la almendra del canime presenta un $80.50 \pm 0.05 \%$ de carbohidratos y un $11.02 \pm 0.03 \%$ de proteínas, en menores proporciones se encuentran la grasa cruda con $3.180 \pm 0.12 \%$ y la fibra con $2.62 \pm 0.049 \%$; lo cual hace de esta semilla un elemento promisorio como alimento para animales. Vale la pena destacar que tanto la almendra como la harina presentan bajo contenido de humedad lo que favorece que no se deterioren los alimentos (Belen et al, 2005). De igual forma se encontró que el contenido de humedad en la harina residual obtenida al extraer el aceite, es mayor que para la almendra $0.0714 \pm 0.0004 \%$ y $0.0830 \pm 0.0026 \%$ respectivamente, lo cual posiblemente se debe a la humedad residual presente en el solvente empleado para la extracción del aceite. En cuanto al análisis de las cenizas los valores obtenidos son muy parecidos tanto para la semilla como para la harina con valores de $2.67 \pm 0.12 \%$ y $2.68 \pm 011 \%$ respectivamente. Adicionalmente, el análisis de proteínas revela que es mayor para la harina con $12.190 \pm 0.020 \%$, que para la almendra con 11.020 $\pm 0.030 \%$, sin embargo ambos presentan buen valor nutricional.

Tabla 2: Análisis proximal de la semilla de canime y de la harina

\begin{tabular}{lcc}
\hline Componente & $\begin{array}{c}\text { Almendra } / \%{ }^{*} \\
x \pm \sigma\end{array}$ & $\begin{array}{c}\text { Harina } / \% \\
x \pm \sigma\end{array}$ \\
\hline Humedad & $0.083 \pm 0.003$ & $0.071 \pm 0.004$ \\
Grasa cruda & $3.180 \pm 0.120$ & \\
Proteínas & $11.020 \pm 0.030$ & $12.190 \pm 0.020$ \\
Cenizas & $2.670 \pm 0.120$ & $2.68 \pm 0.110$ \\
Carbohidratos & $80.500 \pm 0.050$ & \\
Fibra & $2.620 \pm 0.049$ & \\
\hline
\end{tabular}


Los resultados del contenido de minerales fueron analizados utilizando el paquete estadístico el Start Graphic 2:0 y reportados en la tabla 3; donde se muestran las concentraciones de los minerales presentes en la semilla de canime, expresado en $\mathrm{mg} / \mathrm{Kg}$ de muestra, destacandose mayor concentración de potasio y magnesio, le siguen el sodio, zinc y cobre; minerales importantes en el crecimiento y desarrollo de los animales.

Tabla 3: Minerales presentes en la semilla de canime

\begin{tabular}{lc}
\hline \multicolumn{1}{c}{ Minerales } & $\begin{array}{c}\text { muestra } / \mathrm{mg} / \mathrm{Kg} \\
\mathrm{x} \pm \sigma\end{array}$ \\
\hline Potasio & $3.61 \pm 0.02$ \\
Cobre & $0.01 \pm 0.04$ \\
Magnesio & $1.78 \pm 0.12$ \\
Zinc & $0.03 \pm 0.01$ \\
Sodio & $0.11 \pm 0.03$ \\
\hline
\end{tabular}

En la tabla 4, se reportan los valores de porcentajes de extraccion del aceite de la almendra del canime con diferentes solventes, se observa que el mayor porcentaje de rendimiento corresponde al hexano con valor de $3.18 \pm 0.12 \%$ el cual presentó un color amarillo rojizo, le sigue el éter de petróleo con rendimiento de $2.84 \pm 0.13 \%$ el aceite obtenido con este solvente mostró coloración verdosa y el menor porcentaje fue obtenido con acetato de etilo con valor de $2.47 \pm 0.13 \%$; es evidente, que en todos los casos el rendimiento en la extracción del aceite de canime es bastante pobre; razon por el cual no amerita su aislamiento de la almendra.

Tabla 4: Extracción de aceite de la semilla de canime con diferentes solventes

\begin{tabular}{lc}
\hline Solvente & $\begin{array}{c}\text { Rendimiento/ \% } \\
\mathrm{x} \pm \sigma\end{array}$ \\
\hline Acetato de etilo & $2.47 \pm 0.13$ \\
Éter de petróleo & $2.84 \pm 0.13$ \\
$\mathrm{n}$-Hexano & $3.18 \pm 0.12$ \\
\hline
\end{tabular}

En la tabla 5, se muestran los resultados obtenidos de las propiedades fisicoquímicas del aceite extraido por Soxhlet con hexano, como el indice de acidez, considerado como una medida del grado de descomposición del aceite, es un indicador de la presencia de ácidos grasos libres causantes del enranciamiento de los aceites y grasas; por lo tanto este parámetro es usado para indicar el estado y comestibilidad de los mismos, cuyo valor maximo permitido por la industria alimenticia para el aceite refinado es de $0.1 \mathrm{mg} \mathrm{KOH} / \mathrm{g}$ y sin refino $6.6 \mathrm{mg} \mathrm{KOH} / \mathrm{g}$; el grado de acidez obtenido en el aceite en estudio fue de $5.35 \pm 0.11 \%$, se debe tener en cuenta que se trata de un aceite crudo que no ha sido desacidificado y desodorizado en cuyo proceso se eliminan ácidos grasos libres y sustancias volátiles e insaponificables causantes del olor y sabor de los aceites. EL índice de yodo obtenido fue de $69.46 \pm 1.62 \mathrm{cg} \mathrm{l} / \mathrm{g}$, cuyo valor lo clasifica como aceite no secante por ser inferior a $100 \mathrm{cgl} / \mathrm{g}$, el cual favorece su uso para la elaboración de productos cosméticos; el indice de yodo es una escala para determinar el grado de insaturacion de compuestos organicos. El punto de fluidez para el aceite de canime es bastante bajo, proporciona un indicativo de las limitaciones de fluidez que tiene el aceite a bajas temperaturas. El punto de enturbiamiento determinado para el aceite de la semilla de canime es bastante bajo en comparación con otros aceites, el cual resulta favorable ya que indica la baja concentración de parafinas y compuestos interferentes que comienzan a separarse, en forma de cristales cuando el aceite es sometido a un proceso de enfriamiento. 
Tabla 5: Propiedades fisicoquimicas analizadas en el aceite extraído por soxhlet

\begin{tabular}{lc}
\hline Parámetros & Aceite semilla Canime \\
& $x \pm \sigma$ \\
\hline Grado de acidez $(\mathrm{mgKOH} / \mathrm{g})$ & $5.35 \pm 0.11$ \\
Indice de yodo $(\mathrm{cg} \mathrm{l} / \mathrm{g})$ & $69.46 \pm 1.62$ \\
Punto de fluidez & $1.20 \pm 0.10$ \\
Punto de enturbiamiento & $2.20 \pm 0.20$ \\
\hline
\end{tabular}

En cuanto al análisis de la composición quimica del aceite (figura 1) se encontró un total de $96.912 \%$ de acidos grasos, presentando mayor proporción el Vaccenico con $18.112 \%$, Elaídico con $16.031 \%$, linoleico con $15.384 \%$, palmítico con $13.967 \%$, le sigue el 7,10 -octadienoico con $9,781 \%$ behénico con $6.317 \%$, lignocérico con $6.460 \%$ y el resto en menor proporción; en general se puede decir que presenta el $36.602 \%$ de acidos grasos saturados y el $60.31 \%$ insaturados clasificados en monoinsaturados con el $34.143 \%$ y diinsaturados con el $26.167 \%$.

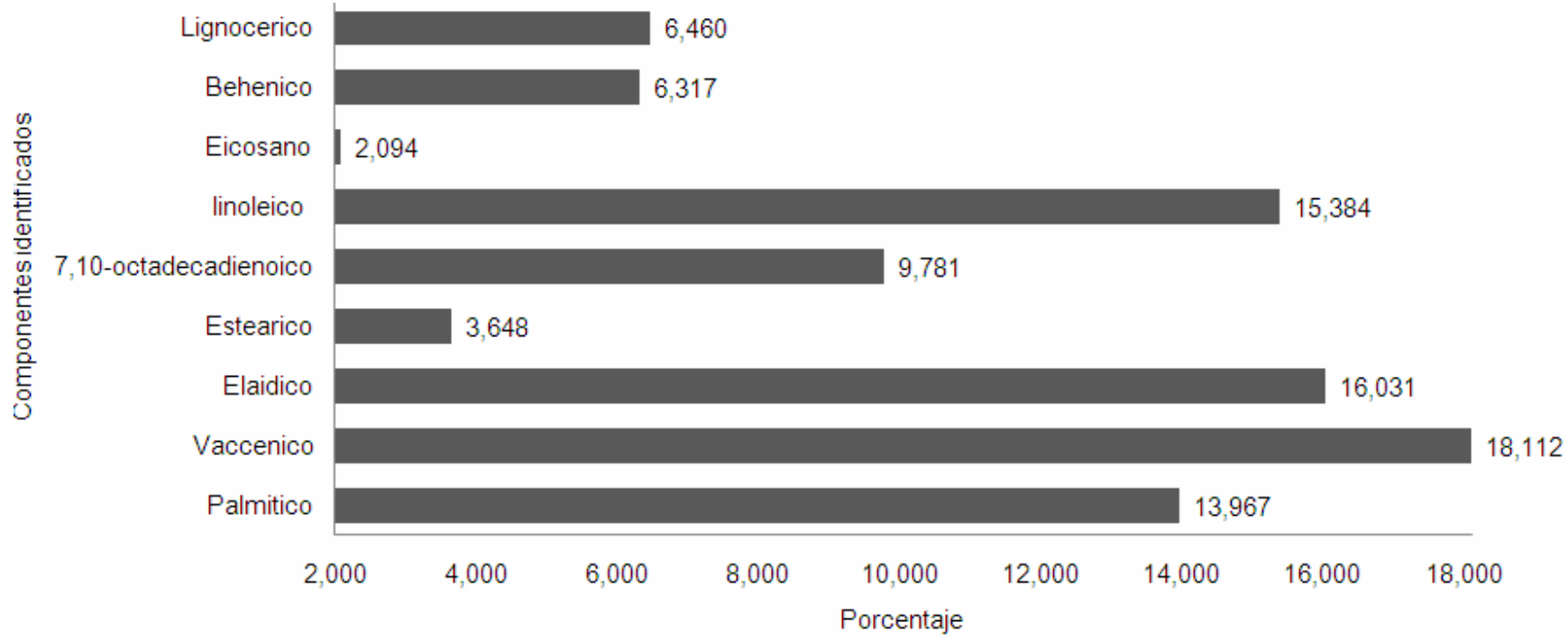

Fig.1: Composición química del aceite de la semilla del Copaifera officinalis (2 -18) \%

Ademas de los acidos grasos tambien se encontró un $2.2798 \%$ de otros compuestos entre ellos, el ácido araquídico y 11-eicosano en mayor proporción, tambien la vitamina $\mathrm{E}$, el esqualeno y butil hidroxitolueno, estos tres últimos son empleados como aditivos, antioxidantes naturales en alimentos, en industrias cosmeticas y farmaceuticas, entre otros usos (figura 2).

La composición quimica del aceite de la semilla de Copaifera officinalis $L$ presenta diferencias con la reportada en el aceite de la semilla del Copaifera langsdorfii (Stupp et al.,2008), la cual contiene: linoleico $(45.34 \%)$, oleico $(30.96 \%)$, palmítico $(12.71 \%)$, estearico $(4.36 \%)$, lignocérico $(2.29 \%)$, behenico $(1.99 \%)$, cis-11-eicosenoico $(1.33 \%)$ y el araquidico $(1.02 \%)$. En ambas especies de Copaifera la mayor proporción está formada por acidos grasos insaturados. 


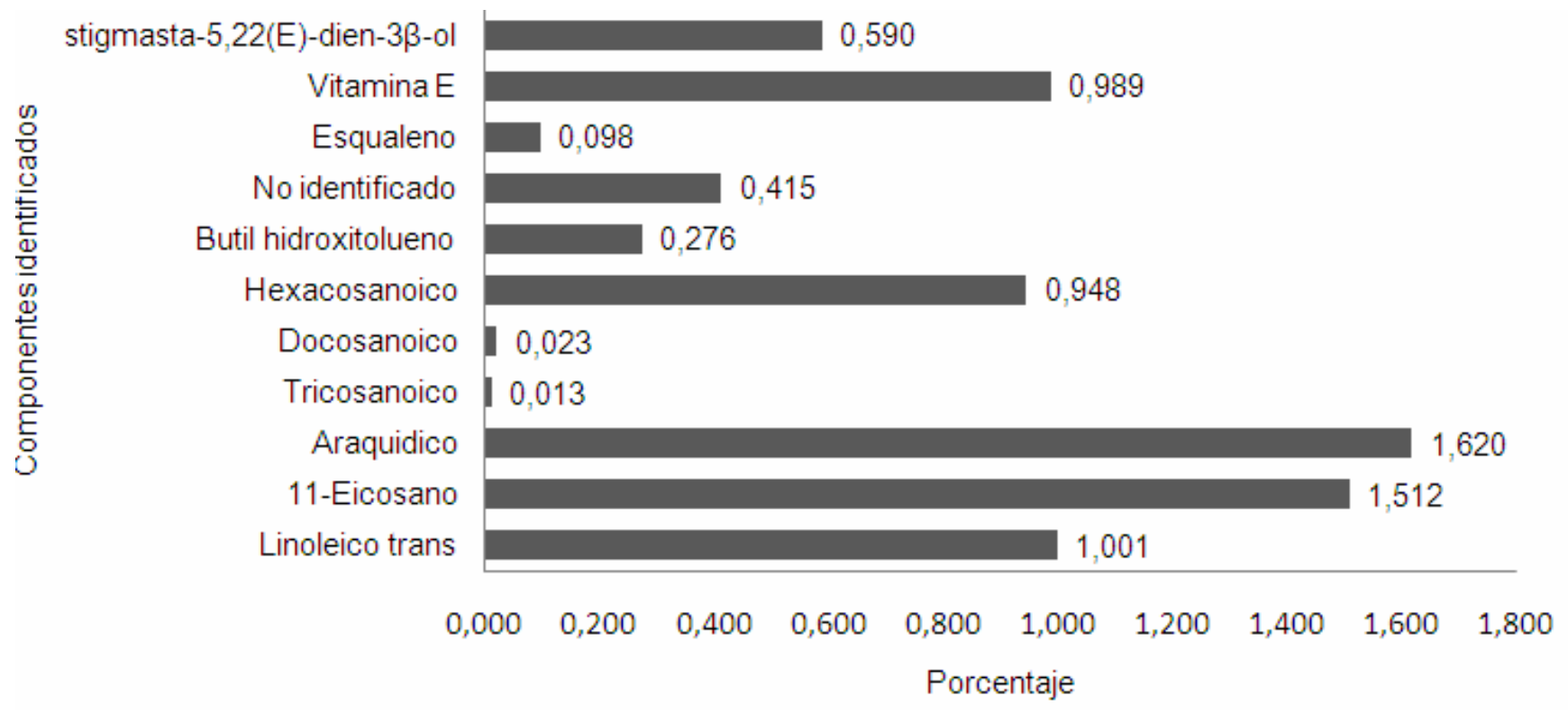

Fig.2: Composición química del aceite de la semilla del Copaifera officinalis (0 - 2) \%

\section{CONCLUSIONES}

Las semillas de canime contienen nutrientes importantes como carbohidratos en su mayor proporción, proteínas, lípidos y minerales; de igual forma la composición química del aceite de esta semilla indicó alto contenido de acidos grasos en su mayor porcentaje insaturados y presencia de vitamina $\mathrm{E}$; estos compuestos son considerados nutrientes principales en la elaboración de alimento para animales, debido a que son las sustancias químicas necesarias para la salud, crecimiento y reproducción del animal; el rendimiento en la extracción del aceite de canime es bastante bajo lo cual no amerita su aislamiento de la almendra; las propiedades fisicoquimicas del aceite indicaron presencia de compuestos orgánicos insaturados; por todo lo anterior se recomienda el aprovechamiento de estas semillas para alimento animal.

\section{AGRADECIMIENTOS}

Al Centro de Investigaciones de la Universidad de Córdoba por financiar el proyecto FCB-05-07 numeral 1120235.

\section{REFERENCIAS}

Alvis, A., C. Velez; H. Villada; M. Rada; Analisis fisico-quimico y morfologico de almidones de yuca y papa y determinacion de la viscosidad de las pastas, Informacion Tecnologica.: 19,19-28 (2008).

Amin, A.M., S.A. Ahma., Y.Y.Yin., N.Yahya., N.Ibrahim; Extraction, purification and characterization of durian (Durio zibethinus) seed gum, Food Hydrocolloids.: 21, 273-279 (2007).

Asogwa, E.U., I.U. Mokwunye., L.E. Yahaya ., A.A. Ajao; Evaluation of cashew nut shell liqiud (CNSL) as a potential natural insecticide against termites (soldiers and workers castes), Research Journal of Applied Sciences.: 2, 939-942 (2007).

ASTM Standard: ASTM D 2500 -99. West Conshohocken: American Society for Testing Materials; Standard Test Method for Cloud Point of Petroleum Products (1999).

ASTM Standard: ASTM D-97-96a. West Conshohocken: American Society for Testing Materials; Standard Test Method for Pour Point of Petroleum Products (1996). 
Chen, F. y otros siete autores; Within-plant distribution and emission of sesquiterpenes from Copaifera officinalis, Plant Physiology and Biochemistry.: 47, 1017-1023 (2009).

Belen, D., y otros cinco autores; Evaluacion fisicoquimica de la semilla y del aceite de corozo (Acrocomia aculeata Jacs), Grasas y aceites.: 56, 317-323 (2005).

Freitas, F. y E.P. Souza; Acaricidal activity of an oleoresinous extract from copaifera reticulate (Leguminoseae: Caesalpinioideae) against larvae of the southern cattle tick, Rhipicephalus (Boophilus) microplus (Acari: Ixiodidae), Veterinary Parasitology.: 147, 150-154 (2007).

Henríquez, C. y otros cinco autores; Characterization of piñon seed (Araucaria araucana (Mol) K. Koch) and the isolated starch from the seed, Food Chemistry.: 107, 592-601 (2008).

Hernandez, C., A. Mieres; Z. Niño; S. Perez; Efecto de la refinacion fisica sobre el aceite dela almendra del corozo (Acrocomia aculeata), Informacion Tecnologica.:18, 59-68 (2007).

Brito, M.V., y otros cinco autores; Copaiba oil effect on urea and creatinine serum levels in rats submitted to kidney ischemia and reperfusion syndrome, Acta Cirurgica Brasileira.: 20, 243-246 (2005).

Matos.N. y otros siete autores; Antineoplasic activity of Copaifera multijuga oil and fractions against ascitic and solid Ehrlich tumor, Journal of Ethnopharmacology.: 119, 179-184 (2008).

Matos.N., C. Moraes., S. Paredes., M. E. Matheus., P. Dias; Antinociceptive activity of Amazonian Copaiba oil, Journal of Ethnopharmacology.: 109, 486-492 (2007).

Sant'Anna, B.M., Paredes, S., Pinto, A.C., Rezende, C; Characterization of Woody Odorant contributors in copaiba oil (Copaifera multijuga Hayne), Journal Brazilian Chemical Society.: 18, 984-989 (2007).

Santos, A.O., y otros cinco autores; Effect of Brazilian copaiba oils on Leishmania amazonensis, Journal of Ethnopharmacology.: 120, 204-208 (2008).

Silva, R., Vieira, G; Sustainability of extraction and production of capaiba (Copaifera multijuga Hayne oleoresin in Manaus, AM, Brazil, Forest Ecology and Management, 256, 282-288 (2008).

Stupp, y otros cinco autores; Characterization and potential uses of Copaifera langsdorfii seeds and seed oil, Bioresource Technology.: 99, 2659-2663 (2008).

Veiga V.F., Rosas, E.C., Carvalho, M.V., Enriquez, M.G., Pinto, A; Chemical composition and antiinflammatory activity of copaiba oils from Copaifera caerensis Huber ex Ducke, Copaifera reticulata Ducke, and Copaifera multijuga Hayne - A comparative study, Journal of Ethnopharmacology.: 112, 248-254 (2007).

Yookyung, K., M. Singh., S. E. Kays; Near-infrared spectroscopic analysis of macronutrients and energy in homogenized meals, Food Chemistry.: 105, 1248-1255 (2007). 